\title{
Sensitivity of Streptococcus pyogenes to sulphamethoxazole, trimethoprim, and cotrimoxazole
}

\author{
E. YOURASSOWSKY, M. P. VANDERLINDEN, AND E. SCHOUTENS \\ Of the Free University of Brussels, the University Hospital Brugmann, Brussels, Belgium
}

SYNOPSIS When tested on Wellcotest (sensitivity test agar, Wellcome) supplemented with lysed horse blood, 59 clinical isolates of beta-haemolytic streptococci, belonging to several serological groups, are all sensitive to sulphamethoxazole, to trimethoprim, and to cotrimoxazole. The results obtained on diagnostic sensitivity test agar (Oxoid) containing lysed horse blood are comparable. When Mueller Hinton agar (Difco) is used, some of these strains are called resistant: this is especially true in the case of sulphamethoxazole.

Furthermore, on Mueller Hinton agar, even supplemented with lysed blood, discrepancies between diameters of inhibition zone and MIC values exist for the three drugs tested, strains with a low MIC being considered resistant by the disc method. Such disagreements are not observed with non-streptococcal control strains.

Streptococcus pyogenes is always sensitive to penicillin G (Matsen, Blazevic, and Chapman, 1969) but can acquire a resistance to erythromycin (Sanders, Foster, and Scott, 1968), lincomycin (Sanders et $a l$ ), and tetracycline (Kuharic, Roberts, and Kirby, 1960). Its sensitivity to sulphonamides is still relatively unknown. A recent WHO report (1968) points out the existence of a group A betahaemolytic streptococcus resistant to sulphonamides. The frequency and geographical location of these strains are not stated, nor the methods used for measuring their sensitivity.

The sensitivity of 59 strains of beta-haemolytic streptococci (belonging to groups A, B, C, and G) to sulphamethoxazole, trimethoprim, and to a combination of these two drugs was studied in vitro on Wellcotest agar enriched with horse blood. The present work reports the results of this study and discusses the choice of culture medium.

\section{Material and Methods}

SENSITIVITY OF Streptococcus pyogenes TO SULPHAMETHOXAZOLE, TRIMETHOPRIM, AND COTRIMOXAZOLE

The bacteria studied were recent isolates from rhinopharyngeal swabs, pus, and blood cultures. Included

Received for publication 16 July 1974. in the survey were 44 strains of group A beta haemolytic streptococci, 10 strains of group B, three strains of group $\mathbf{C}$, and two strains of group $\mathbf{G}$.

Subtyping of group A streptococci was performed at the Institut d'Hygiène et d'Epidémiologie de l'Etat, Brussels, Belgium. Nine strains were untypable, the 35 others fell within 13 different $M$ types among which types 3,12 , and 6 were most frequently found (seven, seven, and five strains respectively).

Minimum inhibitory concentrations (MIC) of the three drugs were determined by the agar dilution method on sensitivity test agar (Wellcotest, Wellcome Reagents Ltd) supplemented with $5 \%$ lysed horse blood. Serial twofold dilutions of sulphamethoxazole and trimethoprim were prepared, starting from initial concentrations of 100 and $10 \mu \mathrm{g}$ per $\mathrm{ml}$ respectively. The sulphamethoxazole-t 1 imethoprim combination was studied in a constant ratio of 20/1. Overnight Mueller Hinton broth cultures were diluted to contain $10^{5}$ organisms per $\mathrm{ml}$. These inocula were spotted with an automatic multipoint inoculator (Dynatech Laboratories, Billingshurst, Sussex) onto the surface of the agar plates (Steers, Foltz, and Graves, 1950). Results were read after overnight incubation at $37^{\circ} \mathrm{C}$ in a $\mathrm{CO}_{2}$ enriched atmosphere (carbon dioxide envelopes, Gas Pak). Hazy zones of growth were ignored when they represented more than an $80 \%$ decrease in bacterial growth (Anderson, 1970). 
Streptococci

Cumulative Percentage of Strains Inhibited at Each Concentration $(\mu \mathrm{g} / \mathrm{ml})$

Sulphamethoxazole

\begin{tabular}{lllllllllll}
\hline 0.024 & 0.48 & 0.09 & 0.19 & 0.39 & 0.78 & 1.56 & 3.12 & 6.25 & 12.5 & 25
\end{tabular}

Trimethoprim

$\begin{array}{llll}0.001 & 0.002 & 0.005 & 0.01\end{array}$

Group A

11

14

17

$19 \quad 33$

53

73

93

100

5

Table Sensitivity of group A (44 strains) and non-group A (15 strains) beta haemolytic streptococci to sulphamethoxazole, trimethoprim, and cotrimoxazole

\section{EFFECT OF COMPOSITION OF MEDIUM}

Included in this control study are 16 strains of group A beta haemolytic streptococci, nine strains of Staphylococcus aureus, nine strains of Escherichia coli, and three strains of Klebsiella pneumoniae.

Their sensitivity to sulphamethoxazole, trimethoprim, and cotrimoxazole was tested by the agar plate dilution method as described above and by the paper disc agar diffusion method (Bauer, Kirby, Sherris, and Turck, 1966), using discs containing $23.75 \mu \mathrm{g}$ sulphamethoxazole, 1.25 $\mu \mathrm{g}$ trimethoprim or a combination of $23.75 \mu \mathrm{g}$ sulphamethoxazole $+1.25 \mu \mathrm{g}$ trimethoprim.

Different culture media were tested, supplemented or not with $5 \%$ lysed horse blood. They included Wellcotest, diagnostic sensitivity test agar (DST) (Oxoïd) and Mueller Hinton agar (Difco).

\section{Results}

\section{SENSITIVITY OF Streptococcus pyogenes}

All 59 strains of beta haemolytic streptococci were sensitive to sulphamethoxazole (MIC $\leqslant 6 \cdot 25 \mu \mathrm{g} / \mathrm{ml}$ ), to trimethoprim (MIC $\leqslant 1.2 \mu \mathrm{g} / \mathrm{ml}$ ), and to cotrimoxazole (MIC $\leqslant 0.78 \mu \mathrm{g}$ sulphamethoxazole $+0.04 \mu \mathrm{g} / \mathrm{ml}$ trimethoprim). Group A strains were generally more sensitive to these drugs than strains of the other groups (table I).

\section{EFFECT OF COMPOSITION OF MEDIUM}

There was no significant difference, depending on the medium utilized, in the MIC values of sulphamethoxazole, for the non-streptococcal organisms (fig 1a). For streptococci, results obtained on Wellcotest were similar whether or not lysed blood was added. The addition of blood slightly improved values obtained on DST but strongly modified those obtained on Mueller Hinton: using the latter, four strains with MIC $\geqslant 31.2 \mu \mathrm{g} / \mathrm{ml}$ were sensitive (MIC $\leqslant 15.6 \mu \mathrm{g} / \mathrm{ml}$ ) when tested on lysed-bloodsupplemented medium.

With few exceptions, mainly observed with Mueller Hinton agar, the nature of the medium used in trimethoprim sensitivity testing little affected the results obtained for all the organisms tested (fig 1b). N

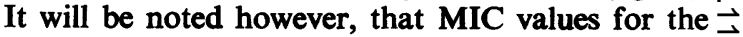
beta haemolytic streptococci were generally slightly o higher on the blood-supplemented Wellcotest than on the other media.

For cotrimoxazole, results, on the whole, correlated well from one medium to another, for all $z$ the organisms tested. Certain strains, however, had an MIC considerably higher when studied on media not enriched with lysed horse blood.

CORRELATION BETWEEN MIC VALUES

DIAMETERS OF INHIBITION ZONES

Many discrepancies were observed with bet haemolytic streptococci between MIC values ant

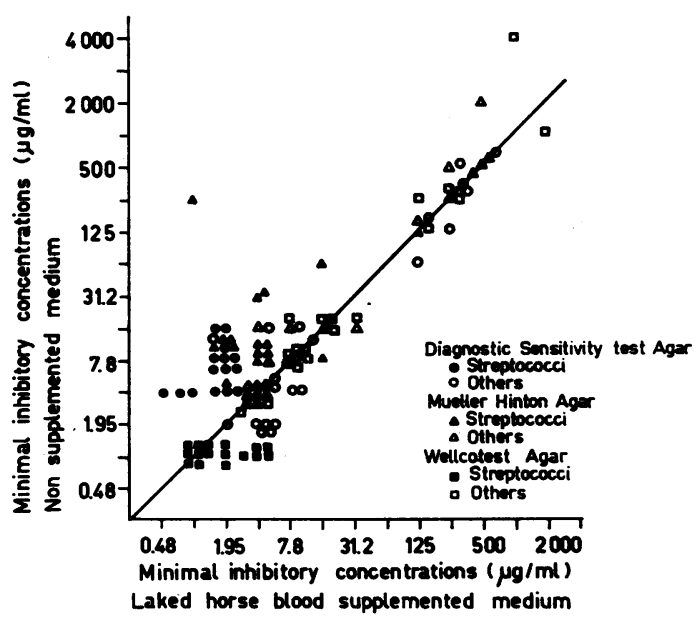

Fig 1a

Fig 1 Comparison of the MIC values of sulphamethoxazole (a), trimethoprim (b), and cotrimoxazole (c) obtained on three different media, enriched or not with lysed horse blood, for streptococci and non-streptococcal organisms. 
Cumulative Percentage of Strains Inhibited at Each Concentration $(\mu \mathrm{g} / \mathrm{ml})$

\begin{tabular}{|c|c|c|c|c|c|c|c|c|c|c|c|c|c|c|c|c|c|}
\hline \multirow[b]{2}{*}{0.02} & \multirow[b]{2}{*}{0.04} & \multirow[b]{2}{*}{0.08} & \multirow[b]{2}{*}{$0 \cdot 16$} & \multirow[b]{2}{*}{$0 \cdot 31$} & \multirow[b]{2}{*}{0.62} & \multirow[b]{2}{*}{$1 \cdot 2$} & \multicolumn{11}{|c|}{ Sulphamethoxazole + Trimethoprim } \\
\hline & & & & & & & $\begin{array}{l}0.024 \\
+ \\
0.001\end{array}$ & $\begin{array}{l}0.048 \\
+ \\
0.002\end{array}$ & $\begin{array}{l}0.09 \\
+ \\
0.005\end{array}$ & $\begin{array}{l}0.19 \\
+ \\
0.01\end{array}$ & $\begin{array}{l}0.39 \\
+ \\
0.02\end{array}$ & $\begin{array}{l}0.78 \\
+ \\
0.04\end{array}$ & $\begin{array}{l}1.56 \\
+ \\
0.08\end{array}$ & $\begin{array}{l}3 \cdot 12 \\
+ \\
0.16\end{array}$ & $\begin{array}{l}6 \cdot 25 \\
+ \\
0.31\end{array}$ & $\begin{array}{l}12.5 \\
+ \\
0.62\end{array}$ & $\begin{array}{l}25 \\
+ \\
1 \cdot 2\end{array}$ \\
\hline 5 & 14 & $\begin{array}{r}30 \\
7\end{array}$ & $\begin{array}{l}76 \\
13\end{array}$ & $\begin{array}{r}100 \\
20\end{array}$ & 74 & 100 & 2 & 5 & 8 & 19 & $\begin{array}{l}63 \\
26\end{array}$ & $\begin{array}{l}100 \\
100\end{array}$ & & & & & \\
\hline
\end{tabular}

Table-continued

diameters of the inhibition zones in trimethoprim and sulphamethoxazole sensitivity testing when using Mueller Hinton agar, supplemented or not with lysed blood (figs $2 b$ and $2 c$ ). For sulphamethoxazole, no medium gave entire satisfaction (fig 2a): DST with lysed blood, and to a lesser extent, Wellcotest with or without lysed blood, gave fewer disagreements.

For the non-streptococcal organisms, discrepancies were rare and only concerned sensitivity testing of Staph. aureus to sulphamethoxazole.

With two exceptions, all disagreements were of the same type: strains with low MIC values were called resistant by the disc method.

\section{Discussion}

Little has been published on the sensitivity of Streptococcus pyogenes to sulphonamides and the few data available are occasionally contradictory. Thus Darrell, Garrod, and Waterworth (1968) report MIC values of sulphamethoxazole ranging from 1 to $16 \mu \mathrm{g}$ per ml whereas Bushby (1969) obtains figures considerably higher, ranging around $100 \mu \mathrm{g}$ per ml. A group of WHO experts (1968) report the 'frequent occurrence' of sulphonamideresistant group A beta haemolytic streptococci; however, many authors recommend sulphonamides as satisfactory alternative agents in prevention (Schultz and Frank, 1958; Stollerman, 1971) or in treatment (Alban, 1965) of beta haemolytic streptococcal infection in subjects sensitive to penicillin. These differences can most likely be attributed to the practical difficulties encountered in the performance and interpretation of sulphonamide sensitivity testing (Harper and Cawston, 1945; McCoy and Pelczar, 1961).

On Wellcotest agar enriched with 5\% laked horse blood all the group A beta haemolytic streptococci that we tested were sensitive to sulphamethoxazole, trimethoprim, and cotrimoxazole; distribution of the strains into several $M$ types excluded the hypo-

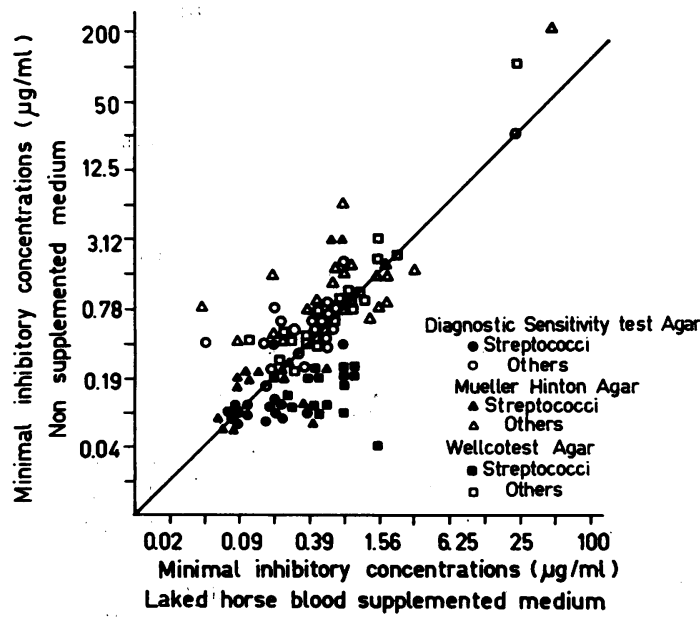

Fig 1b

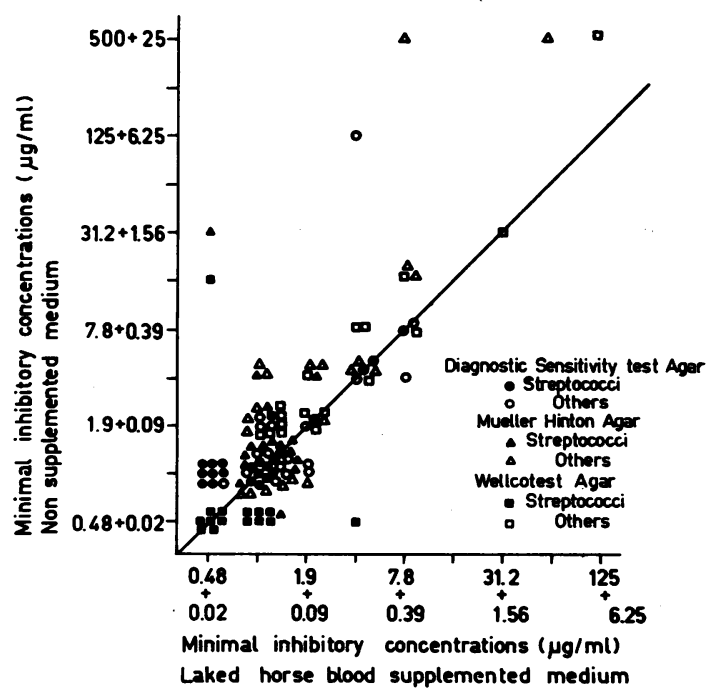

Fig 1c 


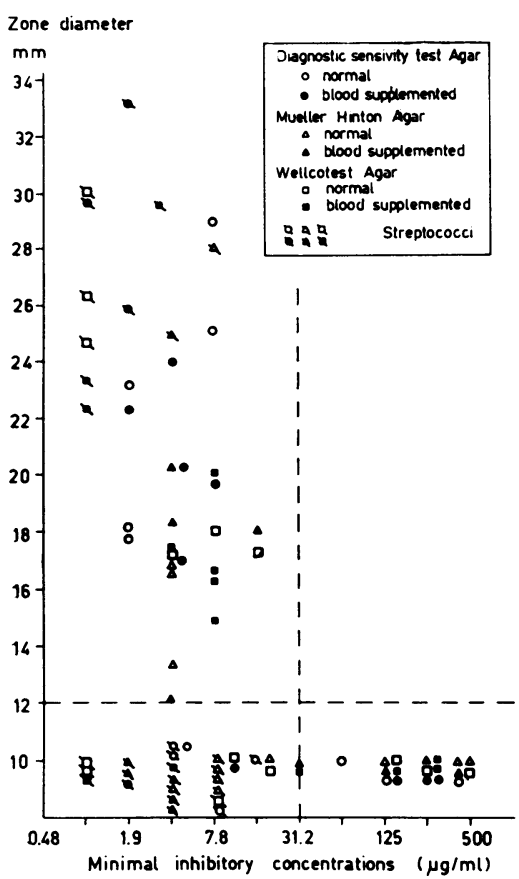

Fig 2a

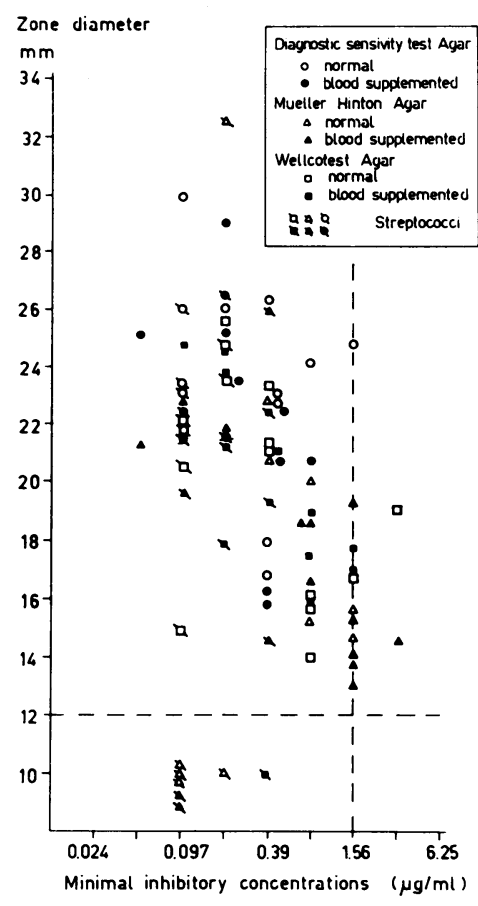

Fig 2b

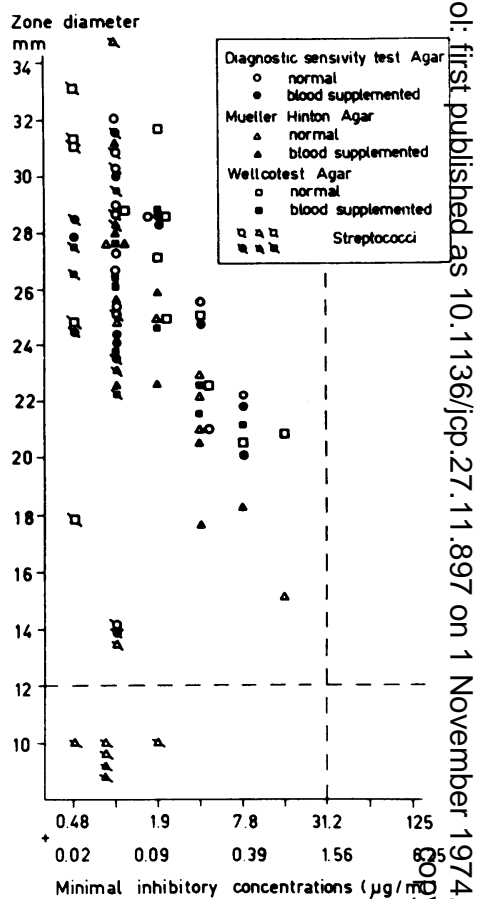

Fig 2c

Fig 2 Relationship between diameters of inhibition zones and MIC values of sulphamethoxazole (a), trimethoprim (b), and cotrimoxazole (c) obtained on three different media, enriched or not with lysed horse blood, for streptococci and non-streptococcal organisms.

thesis of one sensitive strain being prevalent in our institution. Non-group A organisms (belonging to groups B, C, and G) were also highly sensitive although to a slightly lesser degree.

Darrell et al (1968) recommended the addition of lysed horse blood, as for tests of sulphonamide sensitivity (Walker, Philip, Smyth, and McLeod, 1947), to all culture media used in the sensitivity testing of microorganisms to trimethoprim and cotrimoxazole. Furthermore, Dornbusch (1971) showed that Mueller Hinton agar lacking this supplement was not suitable for tests of cotrimoxazole sensitivity. The active factor liberated by horse blood lysis is thymidine phosphorylase (Ferone, Bushby, Burchall, Moore, and Smith, 1973) which inhibits sulphonamide antagonists present in some culture media.

As the choice of Wellcotest agar may appear arbitrary or questionable, two other media frequently recommended for sensitivity testing DST Oxoid and Mueller Hinton Difco-were also evaluated. Furthermore, as a safeguard against erroneous interpretation of the results which would be attributable to a bad batch of medium, the behaviour of non-streptococcal organisms on all three media was also studied.

It can be concluded that no matter which medium is considered, sensitivity testing of Streptococcus pyogenes to sulphamethoxazole, either by determination of the MIC or by the disc method, generally gives better results on media enriched with lysed horse blood. This supplement only slightly modifies results obtained with trimethoprim or cotrimoxazole on the same bacterial species.

Wellcotest and DST agar are satisfactory media for sensitivity testing of beta haemolytic streptococci to sulphamethoxazole, trimethoprim, or cotrimoxazole. In contrast, Mueller Hinton agar, even when supplemented with lysed horse blood, is not suitable for that purpose: strains which are sensitive when tested on the two first media appear resistant when studied on the latter. Moreover, many discrepancies are observed between MIC values and diameters of inhibition zones; however, they only concern 
sensitivity testing of streptococci and are rarely encountered when studying non-streptococcal organisms.

The technical assistance of M. J. Lismont, J. Schoonjans, and J. Dewit is gratefully acknowledged.

We are also grateful to Mrs S. De Maeyer who subtyped the group A streptococci.

\section{References}

Alban, J. (1965). Treatment of $\beta$-hemolytic streptococcal infection. A study of the pediatric use of sulfamethoxazole. Amer. J. Dis. Child., 109, 304-307.

Anderson, T. G. (1970). Testing of susceptibility to antimicrobial agents and assay of antimicrobial agents in body fluids. In Manual of Clinical Microbiology, edited by J. E. Blair, E. H. Lennette, and J. P. Truant, pp. 299-310. American Society for Microbiology, Bethesda, Maryland.

Bauer, A. W., Kirby, W. M. M., Sherris, J. C., and Turck, M. (1966). Antibiotic susceptibility testing by a standardized single disk method. Amer. J. clin. Path., 45, 493-496.

Bushby, S. R. M. (1969). Combined antibacterial action in vitro of trimethoprim and sulphonamides. The in vitro nature of synergy. Postgrad. med. J., Suppl. 45, 10-18.

Darrell, J. H., Garrod, L. P., and Waterworth, P. M. (1968). Trimethoprim: laboratory and clinical studies. J. clin. Path., 21, 202-209.

Dornbusch, K. (1971). Regression line analysis of the synergistic effect for the combination of trimethoprim/sulphamethoxazole. Chemotherapy, 16, 229-238.
Ferone, R., Bushby, S. R. M., Burchall, J. J., Moore, W. B., and Smith, D. (1973). Harper-Cawston factor is thymidine phosphorylase. Abstract A-203-8 ${ }^{\text {th }}$ Intern. Congress of Chemotherapy, Athens.

Harper, G. J., and Cawston, W. C. (1945). The in-vitro determination of the sulphonamide sensitivity of bacteria. J. Path. Bact., 57, 59-66.

Kuharic, H. A., Roberts, C. E., Jr., and Kirby, W. M. M. (1960). Tetracycline resistance of group A beta hemolytic streptococci. J. Amer. med. Ass., 174, 1779-1782.

McCoy, D. W., and Pelczar, M. J., Jr. (1961). Sulphonamide diffusion and inhibition of bacterial growth in agar media. Antimicrob. Agents and Chemother., 811-822.

Matsen, J. M., Blazevic, D. J., and Chapman, S. S. (1969). In vitro susceptibility patterns of beta-hemolytic streptococci. Antimicrob. Agents and Chemother., 485-488.

Sanders, E., Foster, M. T., and Scott, D. (1968). Group A betahemolytic streptococci resistant to erythromycin and lincomycin. New Engl. J. Med., 278, 538-540.

Schultz, I., and Frank, P. F. (1958). The prophylactic use of sulfamethoxypyridazine (Kynex) during a streptococcal epidemic. Amer. J. med. Sci., 236, 779-785.

Steers, E., Foltz, E. L., and Graves, B. S. (1959). An inocula replicating apparatus for routine testing of bacterial susceptibility to antibiotics. Antibiot. Chemother., 9, 307-311.

Stollerman, G. H. (1971). Prophylaxis of streptococcal infection. In Cecil-Loeb Textbook of Medicine, $13^{\text {th }}$ ed. Saunders, Philadelphia.

Walker, N. R., Philip, R., Smyth, M. W., and McLeod, J. W. (1947) Observations on the prevention of bacterial growth by sulfonamides with special reference to the Harper and Cawston effect. J. Path. Bact., 59, 631-645.

World Health Organization (1968). Streptoccal and Staphylococcal infections: report of a WHO Expert Committee, WHO Tech. Rep. Ser., 394, 22. 